\title{
LAS ELECCIONES MEXICANAS DE 1995
}

\author{
Alonso Lujambio*
}

México y Argentina son los dos federalismos latinoamericanos que distribuyen de manera más desgregada sus comicios estatales y locales en la calendarización políticoinstitucional. Por su parte, los otros países federales, Brasil y Venezuela, hacen concurrentes sus procesos para elegir gobernador, cada cuatro años en el primer caso y cada tres en el segundo. En los casos de Brasil y Venezuela, la concurrencia de las elecciones para gobernador impide observar con frecuencia la evolución de las bases de apoyo de los partidos (máxime en ambos países, en donde el sistema de partidos es volátil y/o fragmentado); por el contrario, en los casos argentino y mexicano, la dispersión de elecciones en el tiempo permite (junto con sistemas de partidos mejor estructurados) monitorear le evolución de sus apoyos.

En México se celebraron en 1995 cinco elecciones para gobernador (Jalisco, Yucatán, Guanajuato, Baja California y Michoacán); por otro lado, se celebraron elecciones para renovar el Congreso Estatal y todos los ayuntamientos en 13 estados (Jalisco, Yucatán, Durango, Chihuahua, Baja California, Oaxaca, Zacatecas, Aguascalientes, Chiapas, Puebla, Sinaloa, Tamaulipas y Michoacán) (1) y elecciones para renovar sólamente el Congreso Estatal en dos estados (Veracruz y Tlaxcala).

Las elecciones estatales y locales celebradas en México a lo largo de 1995 están marcadas por cuatro novedades. En primer lugar, cabe subrayar las peculiaridades del contexto. La crisis económica que estalla en diciembre de 1994 (abrupta devaluación del peso, repunte de la inflación, duplicación en seis meses de la tasa de desempleo abierto, caída casi libre de las expectativas futuras, etc.) le planteaba al PRI un complejo dilema a lo largo de todo el año electoral: pagar los costos electorales de la nueva crisis y del draconiano programa de ajuste impulsado por el presidente Zedillo, enfrentando con ello una brutal oleada de votos de castigo, o posponer el ajuste económico para poder, con algún éxito, sortear exitosamente el abultado calendario electoral del año. El presidente Zedillo y su partido optaron por la primera opción, aceptando los costos electorales a corto plazo, y apostando a que para las elecciones legislativas intermedias de 1997 las condiciones económicas mejorarán. Los resultados de este pronóstico optimista están todavía por verse, por supuesto. Era un hecho, de un modo u otro, que se presentaría en 1995

(*) Maestro y Candidato a Doctor en Ciencia Política por la Universidad de Yale. Director del Programa de Ciencia Política en el Instituto Tecnológico Autónomo de México (ITAM).

(1) Recuérdese que México está dividido en 31 estados y un Departamento, el del Distrito Federal. un alud de votos opositores, sobre todo pensando en que, un año antes, el candidato Ernesto Zedillo se presentaba frente al electorado como el hombre que sabía gobernar ("El sabe cómo hacerlo", rezaba la propaganda de Zedillo en las presidenciales de 1994). Pues Zedillo no supo lidiar con la papa caliente que le heredó Carlos Salinas... y así nos fue. Lo único que quedaba por verse desde principios del año era la voluntad de los aparatos priistas locales de respetar los comicios y reconocer la inevitable oleada de votos opositores que se cernía sobre el horizonte.

La segunda novedad de las elecciones se deriva de la primera: como nunca en su larga historia electoral, el PRI recibió un vapuleo, ya cercano a la paliza en algunos rincones del país. El tercer elemento sin precedentes: los procesos electorales (salvo quizá el de Yucatán) fueron limpios y transparentes. Y el cuarto: la competitividad del mercado electoral aumentó al concentrarse el voto opositor en una de las opciones, la de centro-derecha representada por el Partido Acción Nacional (PAN), y al perder presencia el partido opositor de centro-izquierda, el de la Revolución Democrática (PRD).Así pues, tendió a fortalecerse el esquema bipartito PRI-PAN y a debilitarse el tripartidismo PDR-PRIPAN. En una palabra, 1995 fue un año de aceleración para el proceso de transición a la democracia en México.

A continuación se discuten algunas evidencias empíricas sobre el realineamiento de las preferencias electorales en México durante 1995. Primero que nada, cabe subrayar la importancia de un hecho: de las cinco gubernaturas en disputa, el PAN ganó tres (Jalisco, Guanajuato y Baja California) y el PRI dos (Yucatán, con el empleo poco aseado de una cantidad enorme de recursos para el patronazgo clientelar, y Michoacán). Véase el cuadro 1, que despliega los porcentajes obtenidos por el PRI en las cinco elecciones del año bajo estudio. También se presentan ahí los porcentajes obtenidos por el PRI en las últimas tres elecciones, para dar una idea de la evolución de dicho partido en el tiempo. En cuatro de los cinco estados se presentó un formato bipolar PRI-PAN. Sólo en Michoacán se configuró un escenario de tres partidos. Por eso es que, con el 39 por ciento de los votos, el PRI michoacano se pudo llevar la victoria, cuando en Baja California, por ejemplo, perdió la gubernatura con el 42 por ciento de los votos cálidos. En Yucatán, el PRI ganó por 4 puntos porcentuales de ventaja sobre su principal competidor, el PAN. Nótese que, o el PRI perdió elecciones,o las ganó por el estrecho margen o por la división del bloque opositor. La competitividad de las elecciones mexicanas es creciente. 
Cuadro 1

PARTIDO REVOLUCIONARIO INSTITUCIONAL, 1995 APOYO ELECTORAL EN LAS CINCO ELECCIONES PARA GOBERNADOR (PORCENTAJES SOBRE VOTOS VALIDOS)

\begin{tabular}{lcccc}
\hline & $\begin{array}{c}\text { Última } \\
\text { eleccion } \\
\text { para } \\
\text { gobernar }\end{array}$ & $\begin{array}{c}\text { Elecciones } \\
\text { legislativas } \\
1991\end{array}$ & $\begin{array}{c}\text { Elecciones } \\
\text { presidenciales } \\
1994\end{array}$ & $\begin{array}{c}\text { Elecciones } \\
\text { para } \\
\text { gobernador } \\
\mathbf{1 9 9 5}\end{array}$ \\
\hline ESTADO & & & & \\
JALISCO & $57(1988)$ & 63 & 44 & 37 \\
BAJA CALIFORNIA & $38(1989)$ & 46 & 49 & 42 \\
GUANAJUATO & $53(1991)$ & 53 & 54 & 33 \\
MICHOACAN & $50(1992)$ & 54 & 44 & 39 \\
YUCATAN & $59(1993)$ & 62 & 52 & 49 \\
\hline
\end{tabular}

FUENTES: Alonso Lujambio, Federalismo y Congreso en el Cambio Político de México. (México: UNAM, 1995), y Oficina de la Presidencia de la República.

Cuadro 2

PARTIDO ACCION NACIONAL, 1995 APOYO ELECTORAL EN LAS CINCO ELECCIONES PARA GOBERNADOR (PORCENTAJES SOBRE VOTOS VALIDOS)

\begin{tabular}{lcccc}
\hline & $\begin{array}{c}\text { Última } \\
\text { eleccion } \\
\text { para } \\
\text { gobernar }\end{array}$ & $\begin{array}{c}\text { Elecciones } \\
\text { legislativas } \\
1991\end{array}$ & $\begin{array}{c}\text { Elecciones } \\
\text { presidenciales } \\
1994\end{array}$ & $\begin{array}{c}\text { Elecciones } \\
\text { para } \\
\text { gobernador } \\
1995\end{array}$ \\
\hline ESTADO & & & & \\
JALISCO & $48(1988)$ & 45 & 36 & 53 \\
BAJA CALIFORNIA & $48(1989)$ & 45 & 36 & 48 \\
GUANAJUATO & $35(1991)$ & 33 & 29 & 58 \\
MICHOACAN & $7(1992)$ & 9 & 15 & 25 \\
YUCATAN & $37(1993)$ & 36 & 41 & 45 \\
\hline
\end{tabular}

FUENTES: Alonso Lujambio, Federalismo y Congreso en el Cambio Político de México. (México: UNAM, 1995), y Oficina de la Presidencia de la República.

En el cuadro 2 se pude ver el espectacular avance del PAN en esas mismas cinco elecciones para gobernador. Después de una 'pequeña caída en las elecciones presidenciales de 1994, el PAN llega fortalecido a los comicios estatales de 1995.En Jalisco y Guanajuato apaleó. En Michoacán, un estado que desde 1988 venía presentando un claro formato de competencia bipolar PRI-PRD, el PAN logró romper el esquema, robar votos a los dos competidores y más que triplicar sus apoyos si se compara su votación con la obtenida en las últimas elecciones para gobernador, celebrada en 1992.

Cuadro 3

PARTIDO DE LA REVOLUCION DEMOCRATICA, 1995 APOYO ELECTORAL EN LAS CINCO ELECCIONES PARA GOBERNADOR (PORCENTAJES SOBRE VOTOS VALIDOS)

\begin{tabular}{lcccc}
\hline & $\begin{array}{c}\text { Última } \\
\text { eleccion } \\
\text { para } \\
\text { gobernar }\end{array}$ & $\begin{array}{c}\text { Elecciones } \\
\text { legislativas } \\
1991\end{array}$ & $\begin{array}{c}\text { Elecciones } \\
\text { presidenciales } \\
1994\end{array}$ & $\begin{array}{c}\text { Elecciones } \\
\text { para } \\
\text { gobernador } \\
1995\end{array}$ \\
\hline ESTADO & $1(1988)$ & 3 & 7 & 4 \\
JALISCO & $2(1989)$ & 3 & 8 & 4 \\
BAJA CALIFORNIA & $7(1991)$ & 5 & 8 & 7 \\
GUANAJUATO & $35(1992)$ & 31 & 35 & 32 \\
MICHOACAN & $8.1(1993)$ & 0.1 & 3 & 3 \\
YUCATAN & & & & \\
\hline
\end{tabular}

FUENTES: Alonso Lujambio, Federalismo y Congreso en el Cambio Político de México. (México: UNAM, 1995), y Oficina de la Presidencia de la República.
En el cuadro 3 puede constatarse el deterioro electoral de la izquierda mexicana. En ocasiones, su precaria presencia parece empezar a cruzar el umbral, ya de plano, de la inexistencia. En Michoacán, bastión por excelencia del cardenismo, cuna de Cuauhtémoc Cárdenas, el PRD fue incapaz de consolidarse y crecer. El PRI ganó otra vez en Michoacán. Puesta la cosa de otro modo_ de los 16 estados donde se celebraron comicios en 1995, en 12 el PRD vio mermados sus apoyos, en 2 se quedó como estaba, y sólo en dos estados su votación se vió incrementada: en Chiapas (pasó del 32 por ciento al 33 por ciento, si se compara el dato con las presidencias de 1994) y en Zacatecas (pasó del 9 por ciento al 11 por ciento). Nadie en la izquierda mexicana podrá, por supuesto, inflamar entusiasmos con esas cifras.

Otro modo de ver la evolución reciente de las preferencias electorales es la siguiente. En los 16 estados en donde se celebraron comicios durante 1995 vive el 51 por ciento de la población del país. Si agregamos las preferencias en esos estados para las elecciones presidenciales de 1994, encontramos que el PRI obtuvo, en esa mitad del país, el 50 por ciento de los votos, el PAN 26 por ciento, y el PRD 16 por ciento. Si agregamos todos los votos en esos mismos 16 estados para las elecciones locales de 1995, nos encontramos con que el PRI obtiene el 43 por ciento, el PAN 38 por ciento, y el PRD 12 por ciento.Es decir: en la mitad del país, el PAN ya está a 5 puntos porcentuales de distancia del PRI. Otro dato: el 31 por ciento de la población mexicana es gobernada (sea en el nivel municipal o en el gubernatorial o en ambos) por el PAN. Esto habla del avance de la oposición panista, pero también de la posibilidad de generar (después de más de 60 años de unipartidismo) comparación y voto retrospectivo. Esto aumenta también la competitividad del sistema: como elector, no es lo mismo tener como opción la conservación del status quo (el PRI) vs. la incertidumbre total (las oposiciones que nunca han gobernado llegan al gobierno), que tener alguna idea de lo que significan las opciones en el ejercicio de las tareas gubernamentales, por lo menos en los niveles municipal o estatal. El PRI no puede estar más nervioso: a diciembre de 1995, es dable decir que la economía difícilmente repuntará notoriamente para mediados de 1997, cuando se renueve la Cámara de Diputados. ¿Se abre la posibilidad de que el PRI pierda el control mayoritario del Congreso dentro de año y medio? Con prudencia y sin el ánimo de convertirme en futurólogo, yo diría que sí, sobre todo si se piensa que el escándalo nacional e internacional relacionado con los monumentales fraudes de la familia Salinas de Gortari está hundiendo al PRI en el desprestigio. Como si le hiciera falta a esas alturas.

Varios de los dilemas centrales de la transición a la democracia en México tienen que ver con una elección celebrada en 1995 que este ensayo ha ignorado hasta el momento:la de "consejeros ciudadanos" en el Distrito Federal. Efectivamente, a principios de 1995, la Asamblea Legislativa del Distrito Federal aprobó la "Ley de Participación Ciudadana" para elegir una especie de cabildo, por cierto en precarias atribuciones legales, para las 16 "Delegaciones Políticas" en que está dividida la ciudad capital más grande 
del mundo. Recuérdese que desde los años 20 el único elemento "unitario" (no federal) del sistema constitucional mexicano tiene que ver con la existencia de 31 estados federados y un "Departamento" (el Departamento de Distrito Federal).Es por ello que los mexicanos hablamos, con poca inventiva por cierto, de 32 "entidades federativas" y no de 32 "estados federados". Por su puesto, la transición democrática pasa por la democratización del Distrito Federal, es decir, por la creación ya formal del Estado del Valle de México, que deje de ser gobernado por un "Jefe de Departamento" (con enorme poder, por cierto), nombrado por el presidente de la República (sin necesidad de aprobación congresional), y el poder ejecutivo del nuevo estado pase a estar en manos de un gobernador electo por sufragio universal. No puede hablarse de transición democrática en México a cabalidad sí un quinto de la población del país está consitucionalmente impedida para erigir a todos los órganos del gobierno.

\section{Cuadro 4 \\ ¿QUIEN GOBIERNA LAS 30 CIUDADES MAS GRANDES DEL PAIS? \\ (EXCLUIDA EL AREA METROPOLITANA DE LA CIUDAD DE MEXICO) DICIEMBRE DE 1.995}

\begin{tabular}{lrc}
\hline Municipio & Poblacion & $\begin{array}{c}\text { Partido } \\
\text { en el } \\
\text { Gobierno }\end{array}$ \\
\hline 1 GUADALAJARA & $1,650,205$ & PAN \\
2 MONTERREY & $1,069,238$ & PAN \\
3 PUEBLA & $1,057,454$ & PAN \\
4 LEON & 867,920 & PAN \\
5 CIUDAD JUAREZ & 798,499 & PAN \\
6 TIJUANA & 747,381 & PAN \\
7 ZAPOLAN & 712,008 & PAN \\
8 MEXICALI & 601,938 & PAN \\
9 CULIACAN & 601,123 & PAN \\
10 ACAPULCO & 593,212 & PRI \\
11 MERIDA & 556,819 & PAN \\
12 GUADALUPE & 535,560 & PAN \\
13 CHIHUAHUA & 530,783 & PRI \\
14 SAN LUIS POTOSI & 525,733 & PRI \\
15 AGUASCALIENTES & 506,274 & PAN \\
16 MORELIA & 492,901 & PAN \\
17 TOLUCA & 487,612 & PRI \\
18 TORREON & 464,825 & PRI \\
19 QUERETARO & 456,458 & PRI \\
20 HERMOSILLO & 448,966 & PRI \\
21 SAN NICOLAS DE LOS GARZA & 436,603 & PAN \\
22 ALTILLO & 440,920 & PRI \\
23 DURANGO & 413,835 & PT \\
24 VILLAHERMOSA/CENTRO & 386,776 & PRI \\
25 IRAPUATO & 362,915 & PRI \\
26 TLAQUEPAQUE & 339,649 & PAN \\
27 VERACRUZ/PUERTO & 328,607 & PAN \\
28 CELAYA & 310,569 & PRI \\
29 MAZATIAN & 314,345 & PAN \\
30 CAJFME & 311,445 & PRI \\
\hline Fuente: XI CenSo Generalde Población Vivienda & 1,990 & \\
\hline
\end{tabular}

Fuente: XI Censo General de Población y Vivienda, 1.990.

Pues bien, la transición específica del Distrito Federal, que como se verá es todavía más lenta que la del país en su conjunto, está controlada por el PRI del DF. Con mayoría en la Asamblea Legislativa del DF, el PRI aprobó la creacción de la figura del "consejero ciudadano", sujeto de elección popular, pero para no evidenciar la enorme precariedad de sus apoyos electorales en la capital del país, decidió "ciudadanizar" ( $i$ !) la competencia, es decir, imposibilitar a los partidos a presentar candidatos con sus siglas: sólo ciuda- danos "independientes" podrían competir. Así, el 12 de noviembre los habitantes del Distrito Federal no podrían expresar sus preferncias electorales en el sistema de partidos y por supuesto, no podían emitir un voto de castigo en contra del PRI. Así, en la más festiva de las tradiciones hispánicas, el PRI intentó torear a la ciudadanía del Distrito Federal... pero el toro no embistió: sólo el 17 por ciento de la ciudadanía acudió a la opera bufa de la elección de marras, cuando, un año antes, en la elección presidencial de 1994, el 78 por ciento de los ciudadanos del DF acudió a votar. El PRI sigue intentando engañar. Pero ya no engaña a nadie. De una u otra forma, dicho partido fue capaz de evitar otro sonoro triunfo del PAN al salirse él y todos los partidos de la arena electoral capitalina. La "Ley de Participación Ciudadana" aprobada con el voto a favor del PRI en la Asamblea Legislativa del DF posibilitó la construción de este escenario.

El otro dilema también tiene que ver con la necesidad de reformar la Constitución: el art. 41 otorga a los partidos el derecho de participar en elecciones. ¿Por qué una ley secundaria (La de "Participación Ciudadana" del DF) pudo ser aprobada sin ser después declarada inconstitucional "con excepción de las que se refieran a la materia electoral"? Esto es, digamos, la versión mexicana de las cláusulas pinochetistas que aun conserva la Constitución chilena. El PRI, que tiene mayoría absoluta en prácticamente todos los Congresos Estatales, puede votar leyes electorales por donde se puede seguir colando la irregularidad y el abuso, sin que puedan ser declaradas inconstitucionales. Es decir, el PRI en el legislativo esta... por encima de la Constitución! Es claro que uno de los aspectos centrales de la transición mexicana a la democracia tiene que ver con la eventual reforma de los artículos 122 (corresponde al presidente de la República nombrar al Jefe del Departamento del DF) y 105 (no pueden declararse inconstitucionales las leyes electorales, ni la federal ni las 32 locales).

Las elecciones mexicanas de 1995 permiten, sin embargo, ser optimistas sobre la evolución política del país. En 1996, los partidos en el Congreso discutirán una reforma electoral que ya desde ahora bautizan como "definitiva": hacer fraude será más difícil que nunca (tal vez ya imposible) y los medios de comunicación (en particular la televisión) verán reglamentada su apertura a todas las opciones políticas. El PRI ya anunció su disposición a reformar el art. 105 constitucional, de manera que en el futuro las 33 leyes electorales mexicanas (una federal y 32 locales) podrán someterse a juicio constitucional. Las elecciones intermedias de 1997 podrían significar una aceleración profunda, y tal vez ya definitiva, de la transición democrática de México. Un cambio adicional impactará los términos de la competitividad en 1997: me refiero a la redistritación electoral ya anunciada. Efectivamente, los 300 distritos de mayoría simple o relativa de la Cámara de Diputados ( la Asamblea tiene 500 escaños, y 200 se reparten por la vía proporcional a través de listas cerradas y bloqueadas de partidos) no reflejan hoy la composición urbana del país. El trazado de los distritos se hizo hace 16 años, en 1979, con base en una proyección del censo de 1970 . Hoy en día, la población rural está sobrerrepresentada en la Cámara, y la urbana está claramente subrepresentada. La redistribución va a ayudar también a hacer más competitiva la opción del PAN, porque dicho partido tiende a concentrar sus apoyos electorales en las zonas urba- 
nas. Piénsese, por ejemplo, que 11 de las 31 capitales estatales están gobernadas por el PAN. Pero este dato es engañoso, porque las ciudades capitales de los estados no siempre son las más populosas. Véase, para tener una idea de lo que aquí se quiere decir, el cuadro 4 . Ahí puede verse como mientras más grande es una ciudad, más probable es que la gobierne el PAN. Ahora se entiende mejor por qué el PRI quiso sacar a los partidos de la elección del Distrito Federal:. era ya demasiado para el partido hegemónico-en-descomposición reconocer que la capital y las siguientes 9 ciudades más grandes del país eran gobernadas por el PAN. El PRI le tiene pánico a un efecto "bola de nieve" que fortalezca la percepción de que su caída es libre y el ascenso de una fuerza opositora es vertiginosa. Véase en el cuadro que la probabilidad de que una ciudad mexicana de más de 600 mil habitantes sea gobernada por el PAN es del... 100 por ciento. El cuadro sólo enlista a las 30 ciudades más grandes. Pero véase este otro dato: la mitad de las ciudades de más de 100 mil habitantes son gobernadas por el PAN. Así las cosas, cualquier redistritación que quite representación al campo (bastión por excelencia del PRI) y se lo otorgue a la ciudad (ámbito de creciente penetración opositora) benenficiará al Partido Acción Nacional. En 1996, San Luis Potosí, irá a elecciones congresionales. 1996 será la antesala de la elección legislativa intermedia de 1997, blanco sobre el cual todos los partidos afinan ya su puntería: la transición mexicana puede encontrar ahí un claro, y ya definitivo, punto de inflexión. Ya veremos.

\section{RESUMEN}

El artículo analiza las elecciones estatales y locales celebradas en México en 1995. El autor destaca cuatro factores presentes en estos comiciones. En primer lugar, se celebraron en un contexto de crisis económica. En segundo lugar, el PRI cosechó los peores resultados electorales de su historia. En tercer lugar, los procesos electorales (salvo quizá el de Yucatán) fueron limpios y transparentes. Por último, la competitividad del mercado electoral aumentó al concentrarse el voto opositor en una de las opciones, de la centro-derecha representada por el Partido Acción Nacional (PAN), y al perder presencia el partido opositor de centro-iexquierda Partido de la Revolución Democrática (PRD). Así, pues, tendió a fortalecerse el esquema bipartito PRI-PAN y a debilitarse el tripartidismo PRD-PRI-PAN. El autor concluye que 1995 fue un año de aceleración para el proceso de transición a la democracia en México.

\section{ABSTRACT}

This article analyzes the local and federal state elections held in México in 1995. The author mark four principal factors in this elections. First, this elections took place in a context of economical crisis. Second, the PRI had got the worst results in his history. Third, the elections (xcepts maybe in Yucatán) were clean. At last, the election competitiveness increased because of the concentration of the opposing vote in the Partido de Acción Nacional (PAN, centre-right). The centre-leftist Partido de la Revolución Democrática (PRD) losed influence. The author conclusion is that 1995 was a year in wich the transition to democracy go faster. 